\title{
Optimal Service Pricing and Charging Scheduling of an Electric Vehicle Sharing System
}

Xie, Rui; Wei, Wei; Wu, Qiuwei; Ding, Tao; Mei, Shengwei

Published in:

IEEE Transactions on Vehicular Technology

Link to article, DOI:

10.1109/TVT.2019.2950402

Publication date:

2019

Document Version

Peer reviewed version

Link back to DTU Orbit

Citation (APA):

Xie, R., Wei, W., Wu, Q., Ding, T., \& Mei, S. (2019). Optimal Service Pricing and Charging Scheduling of an Electric Vehicle Sharing System. IEEE Transactions on Vehicular Technology, 69(1), 78 - 89.

https://doi.org/10.1109/TVT.2019.2950402

\section{General rights}

Copyright and moral rights for the publications made accessible in the public portal are retained by the authors and/or other copyright owners and it is a condition of accessing publications that users recognise and abide by the legal requirements associated with these rights.

- Users may download and print one copy of any publication from the public portal for the purpose of private study or research.

- You may not further distribute the material or use it for any profit-making activity or commercial gain

- You may freely distribute the URL identifying the publication in the public portal 


\title{
Optimal Service Pricing and Charging Scheduling of an Electric Vehicle Sharing System
}

\author{
Rui Xie, Wei Wei, Senior Member, IEEE, Qiuwei Wu, Senior Member, IEEE, \\ Tao Ding, Senior Member, IEEE, Shengwei Mei, Fellow, IEEE,
}

\begin{abstract}
Electric vehicles (EVs) has tiny environmental impact and will constitute a major mean of urban transportation in the future. Shared EV is quickly becoming a new business model under the sharing economy initiatives, providing easy access for commuters who possess no private cars. In this paper, we consider a carsharing company that owns EVs and some parking lots. Passengers can hire an EV at one parking lot and drive it to another one and pay for the service at a certain price determined by the company. A dedicated EV mobility model is proposed to capture the spatial transportation of energy. Price elasticity is described by a linear demand-price function. The company schedules the charging of unoccupied EVs in each parking lot, aiming at maximizing its profit. Parking lots possess relatively large capacity and have to participate in a distribution power market; energy consumption is paid at the locational marginal price. The decision-making problem of the company is formulated as a bilevel program. The lower level simulates the distribution market clearing, and the upper level represents pricing and charging scheduling problem faced by the company. Starting from a global polyhedral approximation of the power flow model, we develop an equivalent mixed-integer program based on primal-dual optimality condition and integer algebra technique. Case studies demonstrate that the proposed business model can reshape the load profile by shaving the peaking and filling the valley without harming the profit of the company.
\end{abstract}

Index Terms-bilevel program, charging schedules, distribution system, parking lots, pricing, shared electric vehicle

\section{NOMENCLATURE}

\section{A. Parameters}

$T \quad$ Number of time periods.

$\Delta t \quad$ Duration of period.

$N_{S} \quad$ Number of parking lots.

$L_{i j, t} \quad$ Energy consumption of unit vehicle in the trip from parking lot $i$ to $j$ in period $t$.

$a_{i j, t} \quad$ Coefficient of demand function.

$b_{i j, t} \quad$ Coefficient of demand function.

$N_{D} \quad$ Number of buses, excluding the slack bus.

$\rho_{t} \quad$ Electricity price at the slack bus in period $t$.

$\rho_{j, t}^{g} \quad$ Generation cost at bus $j$ in period $t$.

This work is supported in part by the National Natural Science Foundation of China (under grants 51621065 and U1766203) and in part by the Young Elite Scientists Program of CSEE (under grant JLB-2018-95). Corresponding authors: W. Wei and S. Mei.

R. Xie, W. Wei, and S. Mei are with the State Key Laboratory of Power System, Department of Electrical Engineering, Tsinghua University, Beijing, 100084 China. e-mail: (xier17@mails.tsinghua.edu.cn, weiwei04@mails.tsinghua.edu.cn, meishengwei@tsinghua.edu.cn).

Q. Wu is with the Department of Electrical Engineering, Technical University of Denmark, Denmark (Email: qw@elektro.dtu.dk).

T. Ding is with Department of Electrical Engineering, Xi'an Jiaotong University, China (Email: tding15@mail.xjtu.edu.cn). $r_{i j} \quad$ Resistance of the line from $i$ to $j$.

$x_{i j} \quad$ Reactance of the line from $i$ to $j$.

$S(j) \quad$ Set of buses that the power from bus $j$ flows to.

$\sigma(i) \quad$ The bus connected to parking lot $i$.

$\sigma^{-1}(j)$ The parking lot connected to bus $j$.

$N_{i, 0} \quad$ Initial total volume of EVs in parking lot $i$.

$E_{i, 0} \quad$ Initial stored energy of EVs in parking lot $i$.

$E_{\max } \quad$ Stored energy of a fully-charged EV.

$E_{\min } \quad$ Lower bound of the stored energy in an EV.

$\bar{N}_{i} \quad$ Upper bound of EV volume in parking lot $i$.

$\bar{e}_{i} \quad$ Maximum charging energy in a period.

$\bar{p} \quad$ Maximum charging energy of per unit EV in one period.

$p_{j, t}^{d f} \quad$ Fixed active power demand at bus $j$ in period $t$.

$q_{j, t}^{d f} \quad$ Fixed reactive power demand at bus $j$ in period $t$.

$v \quad$ Parameter of linearizing the OPF problem.

$\Delta e_{i} \quad$ Discrete interval of the charging energy.

$N_{B} \quad$ Parameter of discretizing the charging energy.

\section{B. Optimization Variables}

$c_{i j, t} \quad$ Service price of hiring an EV at parking lot $i$ and returning it at parking lot $j$ in period $t$.

$f_{i j, t} \quad$ Volume of EVs traveling from parking lot $i$ to parking lot $j$ in period $t$.

$N_{i, t} \quad$ Total volume of EVs in parking lot $i$ at the end of period $t$.

$E_{i, t} \quad$ Total stored energy in parking lot $i$ at the end of period $t$.

$e_{i, t} \quad$ Charging energy of parking lot $i$ in period $t$.

$p_{0, t}^{g} \quad$ Active power supplied by the slack bus in period $t$.

$p_{j, t}^{g} \quad$ Active generation power at bus $j$ in period $t$.

$q_{j, t}^{g}$

$p_{j, t}^{d}$ Reactive generation power at bus $j$ in period $t$. Active power demand at bus $j$ in period $t$.

$q_{j, t}^{d}$ Reactive power demand at bus $j$ in period $t$.

$P_{i j, t} \quad$ Active power flowing from bus $i$ to bus $j$ at the head node in period $t$.

$Q_{i j, t} \quad$ Reactive power flowing from bus $i$ to bus $j$ at the head node in period $t$.

$v_{i, t} \quad$ Squared voltage magnitude at bus $i$ in period $t$.

$l_{i j, t} \quad$ Squared current from bus $i$ to $j$ in period $t$.

$\mu_{j, t} \quad$ LMP at bus $j$ in period $t$.

$z_{i, t, m}$ Integer variable in discretizing the charging energy at parking lot $i$ in period $t$.

$\alpha_{i, t, m}$ Auxiliary variable in linearizing product terms of LMP and charging energy. 


\section{INTRODUCTION}

$\mathbf{T}$ HE wide deployment of electric vehicles (EVs) and fast-growing distributed renewable generation help cut down the usage of fossil fuels and resolve environmental problems. The sales of EVs keep growing in the past few years [1]. However, some practical issues such as lack of charging facilities and battery degradation problem jeopardize the convenience of EV usage and prevent faster popularization of private EVs [2].

The development of information technology and the advent of smartphone apps. precipitate the boom of sharing economy initiatives, and carsharing is one of the most popular businesses, among others. Shared use of EV provides a broader class of travelers more straightforward access to affordable and clean mobility choices. In a car-hailing service, the user hires a vehicle primarily for a one-way trip. It is different from the so-called ride-sharing [3], in which a solo driver would like to give another passenger a ride. As increasingly more commuters choose not to own a private car for maintenance cost and awareness of environmental issues, carsharing service has thrived amid this new landscape [4].

EV sharing service first appeared in the late 1990s, such as a demonstration program in San Francisco in 1995 [5] and French Praxitele program in 1997 [6]. Nowadays, enabled by smartphone apps., there are many EV sharing programs all over the world, say, Autolib', BlueIndy and EvCard [7].

There are three kinds of car-sharing system: round-way, one-way and free-floating car sharing system [4]. The first one requires clients to return hired vehicles where they depart, which is not convenient. The second one allows returning the vehicle at a different location belonging to the system. In the third one, vehicles can be returned anywhere, even outside the system. Since EVs need recharging, the one-way system is suitable for shared EVs and also comfortable for clients.

The literature related to the one-way EV sharing system can be classified by its perspective, either from the transportation side or the power system side. Research works from the transportation community mainly focus on the relocation problem caused by one-way trips. Since the users can return the car at a different parking lot, there may be accumulation or shortage in some parking lots, and the vehicles in the system need to be relocated to match the demand [8]. There are operatorbased and user-based relocation methods. For operator-based relocation, the vehicles are moved by staffs. In this category, ref. [9] proposes an optimization-trend-simulation decision support system for vehicle relocation in a carsharing system. In [8], an operator-based relocation framework dedicated to EVs is offered, and it can be used to forecast the unbalancing of the system. In user-based relocation, users are encouraged to move vehicles and improve vehicle distribution, which helps to save management costs. In [10], discrete event systems are applied to represent the dynamics of the carsharing system, and a user-based relocation policy is obtained in a rolling horizon framework. The system offers users incentives considering fluctuating demand and parking places in [11], and vehicle allocation schemes are presented. Based on the EV relocation and charging management, the optimal service region design is studied in [12], in which travel needs coverage is maximized under operation cost limits, and robust optimization technique is adopted to deal with uncertainty.

Those from the power system community pay more attention to the charging scheduling in a particular parking lot while neglects the mobility of vehicles. Ref. [13] studies the optimal scheduling of EV charging and discharging, taking into account electricity price, charging time, battery state and age, and tries to earn profit from discharging. In [14], regular and irregular EVs are considered separately, and irregular EVs are described by probabilistic pattern. Ref. [15] focuses on the parking lot in a multi-energy system, where the parking lot appears as the accumulation of EVs, and is regarded as an energy storage unit with uncertainty in the multi-energy system. Ref. [16] considers a parking deck equipped with distributed generation and energy storage, and proposes methods for dayahead and real-time operation. Charging scheduling at several charging stations is modeled as a game in [17], where EV users minimize their waiting time.

Some research works consider the participation of EV parking lots in the electricity market. In [18], a two-stage model is designed to allocate parking lots in distribution systems, where the interaction of parking lots and the electricity market is considered and system costs are minimized. In [19], the behavior of the parking lot in the energy and reserve markets is optimized to maximize the profit, where several kinds of demand response program are addressed respectively. Ref. [20] investigates the equilibrium of the system consisting of energy and reserve markets, EV parking lots, and EV owners. In [21], a bilevel model is proposed for the interaction of parking lots and distribution network, where parking lot operators maximize their profits and distribution system operator minimizes the system cost by optimal power flow.

Some researchers consider charging scheduling outside of parking lot, such as in EV fleet and smart home. In [22], the optimal charging strategy of EV fleet manager is investigated with given service needs information, which is shown to have polynomial complexity. Refs. [23] and [24] present the study of EV operation modes, including grid-to-vehicle and vehicleto-grid, and proposes home-to-vehicle and vehicle-to-home modes. Ref. [25] introduces a method for minimizing the charging cost in smart homes, utilizing vehicle-to-home and vehicle-to-grid modes. Ref. [26] sheds light on the operation of EV battery swapping station considering with solar generation. Nevertheless, in the power system related research, residential or working-place parking lots are individually studied. The mobility of vehicles across different parking lots, which is an important feature of EV sharing system and creates spatial energy transportation, is not treated with enough details.

Wireless charging is an emerging technology for EVs, where charging infrastructure is embedded in the road and EVs are charging when they are traveling [27]. There are a couple of works considering the mobility of EVs in the wireless charging framework. Ref. [28] studies the siting of wireless charging facilities in the transportation network, considering the interaction between traffic flow and charging facility location. According to [29], when the electricity market is based on locational marginal price (LMP), wireless charging 
and EV mobility may have impact on the electricity price. The independence among the electricity and transportation networks through wireless charging of EVs is studied in [30], and short-term operation problem is investigated. In [31], an optimal charging scheduling method is proposed for wirelessly charged electric buses in the public transportation system. Given the technical maturity, this paper still confines to the traditional charging mode.

This paper studies an EV sharing system that consists of a number of parking lots scattering in a certain area. The novelty is twofold.

1) A bilevel optimization model is proposed to help a profit-driven EV sharing company make optimal decisions on service pricing and charging scheduling, taking into account the price elasticity of customers, EV mobility, and demand bidding in a distribution power market. It is different from existing research in two aspects. First, traditional work mainly focuses on the charging scheduling of a single parking lot, and treated every vehicle individually, such as in [13], [14], [16], [18]-[20]. When the mobility of vehicle is taken into account, the arrival rate and the energy demand are usually assumed to follow certain distribution [14], [16], [18], [19]. In an EV sharing system, a customer drives a vehicle from one parking lot and returns it at another one. This mobility is significantly different from what has been considered in the existing literature. In the proposed model, we track the spatial energy transportation among parking lots instead of individual vehicles. The model provides aggregated charging strategy of each parking lot, and leaves single vehicle charging to a self-scheduling problem which has been well studied in the literature, such as [13], [14]. Second, Given the high charging power of EV parking lots, the charging strategy may influence the electricity price, unlike the time-of-use price, which is used in [13]-[15], is exogenous-given constant regardless of the demand. To capture the response of electricity price, we explicitly model the optimal power flow of distribution system from which the accurate locational marginal price is derived.

2) An equivalent mixed-integer quadratic program (MIQP) is developed. To solve such a problem, we perform convex relaxation and global polyhedral approximation of secondorder cones to make the lower-level market clearing problem linear. Then the lower-level problem is replaced by primaldual optimality constraints, and the bilevel problem reduces to a single-level one. Finally, product terms in the objective are linearized via binary expansion, results in a MIQP. To accelerate computation, we propose a warm start procedure to derive a good initial feasible point, which implies a valid upper bound of optimum, and thus the solver can prune unnecessary branches in the early stage.

The rest of this paper is organized as follows: Basic settings and mathematical formulation of the problem is presented in Sect. II. The transformation to a solver compatible MIQP is discussed in Sect. III. Case studies are provided in Sect. IV. In the end, conclusions are drawn in Sect. V.

\section{Problem Formulation}

We first introduce some basic settings and assumptions on the operation of EV sharing system and then presents the optimal power flow (OPF) based distribution market clearing problem. Finally, we pose the pricing and scheduling problem faced by the company.

\section{A. Problem Description and Basic Settings}

We consider a carsharing company that operates a one-way EV sharing system. A typical day is divided into $T$ periods with equal length of $\Delta t$. The company operates $N_{S}$ parking lots where EVs can be charged, hired, and returned. In period $t$, a client hires an EV at parking lot $i$ and returns it at parking lot $j$; the service is charged at price $c_{i j, t}$ announced by the company through a smartphone app.

To model mobility of EVs, let $f_{i j, t} \in \mathbb{R}$ be the volume of vehicles departure from parking lot $i$ to parking lot $j$ in period $t$, and constant $L_{i j, t}$ the energy consumption of unit vehicle in the trip. $L_{i j, t}$ changes across periods due to the time-varying traffic condition. Congestion in the transportation network causes longer travel time as well as energy consumption because of more frequent braking and acceleration. Please be aware that $f_{i j, t}$ is a real number rather than an integer; for example, $f_{i j, t}=0.47$ may represent 47,470 , or an arbitrary integer number of vehicles, depending on the reference base value. We do not model individual vehicles; instead, we consider the aggregated effect. When the total number of EVs is large enough, this setting provides reasonable outcomes while preventing the problem size becoming unmanageable. We assume that all trips are completed in one period; Nonetheless, trips requiring multiple periods can be modeled in the same way.

The volume of clients is influenced by the price, which is approximated by a demand function $f_{i j, t}=a_{i j, t}-b_{i j, t} c_{i j, t}$, where $a_{i j, t}$ and $b_{i j, t}$ are positive coefficients. Price elasticity of demand is a basic concept in economics [32]; the simplest as well as the most widely used one is the linear elasticity. For example, linear price elasticity is used in [33] for modeling carsharing demand, and the impacts of price elasticity parameter are investigated. The parameters can be calibrated from historical data [34]. The logit-based function is also popular to model asymptotical elastic demand. However, the carsharing demand can be zero if the price is too high. Ref. [35] uses a modified logit elastic demand function for pricing, and special techniques are used to form a mixed integer convex problem. Taking the modeling complexity and suitability into account, we adopt the linear demand function in this study.

Let real number $N_{i, t} \in \mathbb{R}$ denote the total volume of EVs in parking lot $i$, at the end of period $t$. Unoccupied EVs are charged in the parking lot. The total stored energy in parking lot $i$ at the end of period $t$ is denoted by $E_{i, t}$, and the charging energy in period $t$ is denoted by $e_{i, t}$. We do not consider the charging of individual vehicles, which is performed through the parking lot self-scheduling and well studied in the existing literature, such as [13], [14].

\section{B. Distribution Network and Market Clearing}

We envision a distribution power market where electricity consumption is charged at the nodal price or locational marginal price (LMP). The market clearing problem relies on 
OPF. As network losses have notable impact on LMP, the renowned direct-current power flow model for a transmission network which is lossless is no longer appropriate. Therefore, we resort to the branch flow model developed in [36] which is shown to be equivalent to the exact alternating-current power flow model [37] for radial distribution networks. Then the OPF-based market clearing problem reads as follows

$$
\begin{array}{ll}
\min & \rho p_{0}^{g}+\sum_{j=1}^{N_{D}} \rho_{j}^{g} p_{j}^{g} \\
\text { s.t. } & P_{i j}-r_{i j} l_{i j}+p_{j}^{g}-p_{j}^{d}=\sum_{k \in S(j)} P_{j k}: \mu_{j}, \forall j \\
& Q_{i j}-x_{i j} l_{i j}+q_{j}^{g}-q_{j}^{d}=\sum_{k \in S(j)} Q_{j k}, \forall j \\
& v_{j}=v_{i}-2\left(r_{i j} P_{i j}+x_{i j} Q_{i j}\right)+\left(r_{i j}^{2}+x_{i j}^{2}\right) l_{i j}, \forall j \\
& \| \begin{array}{c}
2 P_{i j} \\
2 Q_{i j}
\end{array} \\
& l_{i j}-v_{i} \|_{2} \leq l_{i j}+v_{i}, \forall j \\
& \text { variable lower and upper bounds }
\end{array}
$$

where objective function (1a) aims to minimize the operation cost; $\rho$ and $\rho_{j}^{g}$ are the purchase cost at the slack bus and generation cost of unit $j$ respectively; $p_{0}^{g}$ stands for the power delivered from the slack bus, so the first term is paid to the upstream grid, and the second term is the generation cost of local units; (1b) and (1c) are nodal power balance equations; voltage drop along a distribution line is stipulated in (1d); (1e) is the branch flow at the head node of each line; originally, it is a non-convex quadratic equality $P_{i j}^{2}+Q_{i j}^{2}=l_{i j} v_{i}$; by replacing $=$ with $\leq$, the second-order cone (1e) becomes convex; it has been proved that inequality (1e) naturally holds as an equality at the optimal solution under mild conditions [38]; variable lower and upper bounds are imposed in the last constraint. OPF problem (1) is a second-order cone program. The LMP can be extracted from the dual variable $\mu_{j}$ associated with the active power balance equation (1b).

In existing power market studies, linear power flow models are used for market clearing, such as the renowned directcurrent power flow model [39] and the linearized branch flow model [40], [41], owing to the computational superiority of linear program. However, the above linear power flow models neglects network losses and inevitably involve approximation error, especially for distribution systems where lines have a large resistance to reactance ratio. So we resort to the branch flow model based formulation (1), which better captures the operating characteristics of distribution systems, for calculating electricity prices. Using convex relaxation of OPF models for distribution market clearing has been discussed in [42] and [43]. Nonetheless, the market clearing problem will be incorporated in a bilevel program, yielding a more challenging optimization problem.

\section{Operation of the EV Sharing System}

Operating the EV sharing system entails pricing every single trip between any two parking lots and the aggregated charging strategy of each parking lot. The problem is cast as follows

$$
\begin{array}{ll}
\max & \sum_{t=1}^{T} \sum_{i=1}^{N_{S}} \sum_{j=1}^{N_{S}} c_{i j, t} f_{i j, t}-\sum_{t=1}^{T} \sum_{i=1}^{N_{S}} \mu_{\sigma(i), t} e_{i, t} \\
\text { s.t. } \quad f_{i j, t}=a_{i j, t}-b_{i j, t} c_{i j, t}, \forall i, \forall j, \forall t & \\
& N_{i, t}=N_{i, 0}+\sum_{s=1}^{t} \sum_{j=1}^{N_{S}}\left(f_{j i, s}-f_{i j, s}\right), \forall i, \forall t \\
& E_{i, t}=E_{i, 0}+\sum_{s=1}^{t} e_{i, s}-\sum_{s=1}^{t} \sum_{j=1}^{N_{S}} E_{\max } f_{i j, s} \\
& +\sum_{s=1}^{t} \sum_{j=1}^{N_{S}}\left(E_{\max }-L_{j i, s}\right) f_{j i, s}, \forall i, \forall t \\
& E_{\text {max }} \sum_{j=1}^{N_{S}} f_{i j, t}+E_{\min }\left(N_{i, t}-\sum_{j=1}^{N_{S}} f_{i j, t}\right) \leq E_{i, t-1} \\
& E_{i, t} \leq E_{\text {max }} N_{i, t}, \forall i, \forall t \\
& N_{i, T}=N_{i, 0}, E_{i, T}=E_{i, 0}, \forall i \\
& f_{i j, t} \geq 0, c_{i j, t} \geq 0, \forall i, \forall j, \forall t \\
& 0 \leq N_{i, t} \leq \bar{N}_{i}, \quad e_{i, t} \geq 0, \forall i, \forall t \\
& e_{i, t} \leq \bar{e}_{i}, e_{i, t} \leq \bar{p} N_{i, t}, \forall i, \forall t \\
\mu_{\sigma(i), t} \text { is determined from }(1)
\end{array}
$$

In objective function (2a), the first item is the total income for renting EVs, and the second item is the charging cost paid to the distribution power market. The profit is to be maximized. The deprecation cost of EV is not considered in the objective function, because the problem pertains to the daily operation whose timescale is much shorter than that of battery degradation. The deprecation cost is also neglected in daily operation related work in [13], [15], [16]. Nevertheless, the deprecation cost of EV can be considered in the objective function via a linear term in $f_{i j, t}$.

Constraint (2b) depicts the elasticity of clients. Constraint (2c) represents the volume of EVs in parking lot $i$ at the end of period $t$, considering those leaving to/arriving from other parking lots. Denote by $E_{\max }$ the stored energy of a fully-charged $\mathrm{EV}$, and $L_{j i, t}$ the energy consumption of travelling from parking lot $j$ to parking lot $i$; because of time-varying traffic condition, such an energy consumption could be different in each time period. With these notations, $E_{\text {max }} f_{i j, s}$ is the energy taken away by EVs setting out for parking lot $j, L_{j i, s} f_{j i, s}$ is the energy used in travelling, and $\left(E_{\max }-L_{j i, s}\right) f_{j i, s}$ is the energy brought by EVs coming from parking lot $j$; therefore, constraint (2d) characterizes the total energy stored in parking lot $i$ at the end of period $t$; constraints (2c) and (2d) constitute the EV mobility model. It captures the spatial transportation of energy.

As a consequence of the aggregated modeling paradigm, we do not distinguish individual vehicles that are unoccupied in the parking lot. For the ease of model setup, we assume that an EV is available to use only it is fully charged, i.e., the battery storage reaches $E_{\max }$. This assumption brings great convenience to practical operation and theoretical analysis. 
From the practical perspective, if a vehicle is allowed to leave at any time regardless of its charging status, the chargingdischarging cycle may increase during a certain period, which harms the life of the battery. Under this assumption, there is no need to check if the energy left in the battery is sufficient for the specific travel. From the modeling perspective, the spatial transportation of energy can be conveniently expressed in (2d). Without this assumption, it is non-trivial to estimate the spatial energy flow among the parking lots.

Denote by $E_{\min }$ the lower bound of the stored energy in an EV. If some vehicles are needed in period $t$, they must take away total energy with the amount of $E_{\max } \sum_{j=1}^{N_{S}} f_{i j, t}$, and the remaining vehicles in the parking lots possess total energy with the amount of at least $E_{\min }\left(N_{i, t}-\sum_{j=1}^{N_{S}} f_{i j, t}\right)$. Constraint (2e) means that the total stored energy at the end of period $t-1$ is adequate for ensuring that adequate vehicles are standing by at the beginning of period $t$. Constraint (2f) is the upper bound of the total stored energy. Constraints (2e) and (2f) guarantee the energy adequacy in each charging station.

Constraint (2g) imposes equal operating states at the beginning and end of each day in order to complete a daily cycle. Constraint (2h) stipulates non-negativity on service price $c_{i j, t}$, customer volume $f_{i j, t}$, maximal $\mathrm{EV}$ volume $N_{i, t}$ restricted by the total number of charging piles/slots in the parking lot, and maximal charging power depending on the transformer capacity of the parking lot, and maximum charging rate of EV batteries. Because EV charging accounts for a high fraction of distribution system load and impacts nodal electricity price, in order to maximize profit, the company has to take the LMP reaction from the distribution market into account, which is stated in constraint (2i). Denote by $\sigma(i)$ the bus in the distribution system where parking lot $i$ is connected, the LMP $\mu_{\sigma(i), t}$ is determined from the dual variable of OPF problem (1), where charging demand $e_{i, t}$ is added with the original fixed demand $p_{\sigma(i) t}^{d f}$, i.e.:

$$
p_{\sigma(i), t}^{d}=p_{\sigma(i), t}^{d f}+e_{i, t} / \Delta t, \forall i, \forall t
$$

For buses without a parking lot connection, the active and reactive power demands are known constants. For notation brevity, time label $t$ is omitted in OPF problem (1) without causing confusion.

Formulation (2) is actually a bilevel optimization problem. The lower level simulates distribution market clearing, and the upper level represents pricing and charging scheduling problem faced by the company.

\section{AN EQUIVALENT MIQP}

The difficulty of solving problem (2) is twofold: nonlinear item $\mu_{\sigma(i), t} e_{i, t}$ in the objective function and LMP constraint (2i). Furthermore, $\mu_{\sigma(i), t}$ is a dual variable determined by the OPF problem, intensifying the computational challenge. In this section, problem (2) will be approximated by an MIQP, which can be globally solved by commercial solvers. First, the outer polyhedral approximation is applied to second-order cones, transforming the OPF problem into a linear program, which is then substituted by its primal-dual optimality condition; finally, product $\mu_{\sigma(i), t} e_{i, t}$ as well as remaining nonlinearity will be linearized via integer programming technique.

\section{A. Linearizing the OPF Problem}

Solving a bilevel optimization problem often entails replacing the lower-level problem with its optimality condition, usually the KKT condition, leading to a mathematical program with complementarity constraints. Complementarity constraints are hard to tackle not only because they are nonconvex, but also due to their intrinsic numeric instability [44]. In power system applications, economy is the primary concern and global optimality is always desired. In most existing power market studies, the market clearing problem is linear. The computational advantage brought by the linear market clearing problem is that when it is replaced by the corresponding KKT optimality condition, the linear complementarity constraints can be linearized by the well-known big-M method [45]; then the problem can be further reformulated as a mixed-integer linear program that can be globally solved by off-the-shelf solvers. However, OPF problem (1) for distribution system is nonlinear, albeit convex, and its KKT optimality condition entails second-order cone complementarity constraints, preventing further linearization. In this regard, we resort to linearizing the OPF problem first, and then standard optimality conditions can be applied.

In problem (1), the only nonlinearity originates from the second-order cone constraint (1e). We adopt the global polyhedral approximation technique proposed in [46]. Compared with other linear OPF models, the maximum approximation error over the entire power flow feasible region can be arbitrarily small whenever the SOCP relaxation model (1) is exact, i.e., equality holds for (1e) at the optimal solution.

The technique in [46] is developed for standard secondorder cones in $\mathbb{R}^{3}$ with the form of $\sqrt{x_{1}^{2}+x_{2}^{2}} \leq x_{3}$. To apply this technique, constraint (1e) is decomposed into a pair of standard second-order cones

$$
\begin{gathered}
\sqrt{\left(2 P_{i j}\right)^{2}+\left(2 Q_{i j}\right)^{2}} \leq W_{i j} \\
\sqrt{W_{i j}^{2}+\left(l_{i j}-v_{i}\right)^{2}} \leq l_{i j}+v_{i}
\end{gathered}
$$

Applying the global polyhedral approximation technique in [46] to (4a) and (4b), respectively, we obtain two sets of linear constraints

$$
\begin{aligned}
& \left\{\begin{array}{l}
\xi_{i j, 1}^{0} \geq 2 P_{i j}, \xi_{i j, 1}^{0} \geq-2 P_{i j} \\
\eta_{i j, 1}^{0} \geq 2 Q_{i j}, \eta_{i j, 1}^{0} \geq-2 Q_{i j}
\end{array}\right. \\
& \left\{\begin{array}{l}
\xi_{i j, 1}^{k}=\cos \left(\frac{\pi}{2^{k+1}}\right) \xi_{i j, 1}^{k-1}+\sin \left(\frac{\pi}{2^{k+1}}\right) \eta_{i j, 1}^{k-1} \\
\eta_{i j, 1}^{k} \geq-\sin \left(\frac{\pi}{2^{k+1}}\right) \xi_{i j, 1}^{k-1}+\cos \left(\frac{\pi}{2^{k+1}}\right) \eta_{i j, 1}^{k-1} \\
\eta_{i j, 1}^{k} \geq \sin \left(\frac{\pi}{2^{k+1}}\right) \xi_{i j, 1}^{k-1}-\cos \left(\frac{\pi}{2^{k+1}}\right) \eta_{i j, 1}^{k-1}
\end{array}\right. \\
& \left\{\begin{array}{l}
\xi_{i j, 1}^{v} \leq W_{i j} \\
\eta_{i j, 1}^{v} \leq \tan \left(\frac{\pi}{2^{v+1}}\right) \xi_{i j, 1}^{v}
\end{array}\right.
\end{aligned}
$$




$$
\begin{aligned}
& \left\{\begin{array}{l}
\xi_{i j, 2}^{0} \geq W_{i j}, \xi_{i j, 2}^{0} \geq-W_{i j} \\
\eta_{i j, 2}^{0} \geq l_{i j}-v_{i}, \eta_{i j, 2}^{0} \geq-\left(l_{i j}-v_{i}\right)
\end{array}\right. \\
& \left\{\begin{array}{l}
\xi_{i j, 2}^{k}=\cos \left(\frac{\pi}{2^{k+1}}\right) \xi_{i j, 2}^{k-1}+\sin \left(\frac{\pi}{2^{k+1}}\right) \eta_{i j, 2}^{k-1} \\
\eta_{i j, 2}^{k} \geq-\sin \left(\frac{\pi}{2^{k+1}}\right) \xi_{i j, 2}^{k-1}+\cos \left(\frac{\pi}{2^{k+1}}\right) \eta_{i j, 2}^{k-1} \\
\eta_{i j, 2}^{k} \geq \sin \left(\frac{\pi}{2^{k+1}}\right) \xi_{i j, 2}^{k-1}-\cos \left(\frac{\pi}{2^{k+1}}\right) \eta_{i j, 2}^{k-1}
\end{array}\right. \\
& \left\{\begin{array}{l}
\xi_{i j, 2}^{v} \leq l_{i j}+v_{i} \\
\eta_{i j, 2}^{v} \leq \tan \left(\frac{\pi}{2^{v+1}}\right) \xi_{i j, 2}^{v}
\end{array}\right.
\end{aligned}
$$

where $\xi_{i j, 1}^{k}, \eta_{i j, 1}^{k}, \xi_{i j, 2}^{k}, \eta_{i j, 2}^{k}, k=0,1, \cdots, v$ are auxiliary variables; $v$ is a positive integer, which is used to adjust approximation accuracy.

The basic idea behind (5) and (6) is to create a rotation mapping which preserves the norm of the complex number $\xi^{k}+\mathrm{j} \eta^{k}$. Once the angle of the complex number is close to 0 , the real part is approximately the norm. According to [46], if the original constraint (1e) is satisfied, then (5) and (6) must hold; conversely, if (5) and (6) hold, then

$$
\left\|\begin{array}{c}
2 P_{i j} \\
2 Q_{i j} \\
l_{i j}-v_{i}
\end{array}\right\|_{2} \leq(1+\delta(v))^{2}\left(l_{i j}+v_{i}\right)
$$

is satisfied, where

$$
\delta(v)=\frac{1}{\cos \left(\frac{\pi}{2^{v+1}}\right)}-1=O\left(\frac{1}{4^{v}}\right)
$$

Therefore, (5)-(6) provide a global outer approximation of second-order cones defined in (4a)-(4b). Equation (8) provides an estimation on the approximation error bound, which decreases rapidly with the growth of $v$.

In view of this relation, we can replace (1e) with (5)-(6), and thus problem (1) becomes a linear program

$$
\begin{aligned}
& \min \rho p_{0}^{g}+\sum_{j=1}^{N_{D}} \rho_{j}^{g} p_{j}^{g} \\
& \text { s.t. }(1 b)-(1 d),(5)-(6)
\end{aligned}
$$

variable lower and upper bounds

whose compact form can be written as

$$
\begin{aligned}
\min _{y} & C^{T} y \\
\text { s.t. } & A_{1} y=p^{d}: \mu \\
& A_{2} y \geq B_{2}
\end{aligned}
$$

where vector $y$ encapsulates physical variables $p_{j}^{g}, q_{j}^{g}, v_{j}, P_{i j}$, $Q_{i j}, l_{i j}$ and auxiliary variables $\xi_{i j, 1}^{k}, \eta_{i j, 1}^{k}, \xi_{i j, 2}^{k}, \eta_{i j, 2}^{k}$, and matrices $C, A_{1}, A_{2}, p^{d}$ and $B_{2}$ are constant coefficients. (10b) corresponds to nodal active power balancing condition, where charging demand of parking lots is merged in $p^{d}$ via equation (3). $\mu$ is the dual variable associated with (10b), and its $j$-th entry is the LMP at bus $j$. All other equality and inequality constraints are abstracted in (10c).
To eliminate the min operator in constraint (2i), LP (10) will be replaced by its optimality condition. There are two major optimality conditions of LP, namely KKT optimality condition and primal-dual optimality condition, which are equivalent to each other for LPs. We choose primal-dual optimality condition [47]-[49], because a large number of inequalities are used in polyhedral approximation (5)-(6); if the KKT condition is used, each inequality will introduce a complementarity and slackness constraint in the KKT condition, prohibiting an efficient solution, although they can be linearized in a somehow different way.

To explain the primal-dual optimality condition, the dual LP of problem (10) is

$$
\begin{aligned}
\max _{\mu, \gamma} & \mu^{T} p^{d}+\gamma^{T} B_{2} \\
\text { s.t. } & A_{1}^{T} \mu+A_{2}^{T} \gamma=C \\
& \gamma \geq 0
\end{aligned}
$$

Because strong duality always holds for LP, objective functions (10a) and (11a) take the same value at the optimal solution. Therefore, primal problem (10) and dual problem (11) achieve optimum if and only if variables $(y, \mu, \gamma)$ satisfy

$$
\begin{aligned}
& A_{1} y=p^{d}, A_{2} y \geq B_{2} \\
& A_{1}^{T} \mu+A_{2}^{T} \gamma=C, \gamma \geq 0 \\
& C^{T} y=\mu^{T} p^{d}+\gamma^{T} B_{2}
\end{aligned}
$$

where (12a) and (12b) represent primal and dual feasibility constraints, respectively, and (12c) stipulates strong duality. Taking into account (3) and time index $t$, constraint (2i) in (2) can be replaced with the following optimality condition

$$
\begin{aligned}
& A_{1} y_{t}=p_{t}^{d f}+\frac{e_{\sigma^{-1}, t}}{\Delta t}, A_{2} y_{t} \geq B_{2, t} \\
& A_{1}^{T} \mu_{t}+A_{2}^{T} \gamma_{t}=C, \gamma_{t} \geq 0 \\
& C^{T} y_{t}=\left(p_{t}^{d f}+\frac{e_{\sigma^{-1}, t}}{\Delta t}\right)^{T} \mu_{t}+B_{2, t}^{T} \gamma_{t}
\end{aligned}
$$

for $t=1, \cdots, T$, where $p_{t}^{d f}$ is the vector of fixed active and reactive loads in period $t$, and $e_{\sigma^{-1}, t}$ is the vector of parking lot charing demands in period $t$.

\section{B. Linearizing Product Terms}

Through above procedures, problem (2) is reduced to a nonlinear program; bilinear terms $\mu_{\sigma(i), t} e_{i, t}$ and $e_{\sigma^{-1}(j), t} \mu_{j, t}$ rest in objective function (2a) and constraint (13c). Such items can be approximated by a so-called binary expansion method [50]. To this end, we first discretize $e_{i, t}$ as follows

$$
e_{i, t}=\Delta e_{i} \cdot \sum_{m=0}^{N_{B}-1} 2^{m} z_{i, t, m}, \Delta e_{i}=\frac{\bar{e}_{i}}{2^{N_{B}}}
$$

where $z_{i, t, m} \in\{0,1\}$ is a binary variable. In equation (14), the possible value of continuous variable $e_{i, t} \in\left[0, \bar{e}_{i}\right]$ is approximated by $2^{N_{B}}$ uniformly distributed candidates; or in other words, the required number of binary variables is a 
logarithmic function in the number of breakpoints. With (14), bilinear term $\mu_{\sigma(i), t} e_{i, t}$ can be expressed via linear functions

$$
\begin{gathered}
\mu_{\sigma(i), t} e_{i, t}=\Delta e_{i} \sum_{m=0}^{N_{B}-1} 2^{m} \alpha_{i, t, m} \\
0 \leq \alpha_{i, t, m} \leq M z_{i, t, m} \\
0 \leq \mu_{\sigma(i), t}-\alpha_{i, t, m} \leq M\left(1-z_{i, t, m}\right) \\
e_{\sigma^{-1}(j), t} \mu_{j, t}=\Delta e_{\sigma^{-1}(j)} \sum_{m=0}^{N_{B}-1} 2^{m} \alpha_{\sigma^{-1}(j), t, m} \\
z_{i, t, m} \in\{0,1\}, \forall i, \forall t, \forall m
\end{gathered}
$$

where $M$ is a large enough constant. To see their equivalence, if $z_{i, t, m}=1$, (15c) enforces $\alpha_{i, t, m}=\mu_{\sigma(i), t}$, and (15b) is redundant; otherwise, if $z_{i, t, m}=0$, (15b) dictates $\alpha_{i, t, m}=0$, and (15c) is redundant. Hence, relation $\alpha_{i, t, m}=z_{i, t, m} \mu_{\sigma(i), t}$ holds. Substituting it into (15a), it is a natural consequence of multiplying both sides of (14) with $\mu_{\sigma(i), t}$; nevertheless, the right-hand side of (15a) and constraints (15b)-(15e) are all linear. The same can be applied to bilinear term $e_{\sigma^{-1}(j), t} \mu_{j, t}$.

\section{Final MIQP}

Eliminating $c_{i j, t}$ from (2b) and substituting it into objective function (2a), the first term remains convex. Performing all procedures presented in previous subsection, we obtain the final MIQP as follows

$$
\begin{aligned}
\max & \sum_{t=1}^{T} \sum_{i=1}^{N_{S}} \sum_{j=1}^{N_{S}}\left(-\frac{1}{b_{i j, t}} f_{i j, t}^{2}+\frac{a_{i j, t}}{b_{i j, t}} f_{i j, t}\right) \\
& -\sum_{t=1}^{T} \sum_{i=1}^{N_{S}} \Delta e_{i} \sum_{m=0}^{N_{B}-1} 2^{m} \alpha_{i, t, m} \\
\text { s.t. } & (2 b)-(2 h),(13 a),(13 b) \\
& (14),(15 b),(15 c),(15 e) \\
& C^{T} y_{t}=B_{2, t}^{T} \gamma_{t}+\left(p_{t}^{d f}\right)^{T} \mu_{t} \\
& +\frac{1}{\Delta t} \sum_{j} \Delta e_{\sigma^{-1}(j)} \sum_{m=0}^{N_{B}-1} 2^{m} \alpha_{\sigma^{-1}(j), t, m}
\end{aligned}
$$

Problem (16) can be solved by commercial solvers.

To expedite computation, we propose an initiation procedure to derive a good starting point which is feasible in (16), because a feasible solution can imply a valid upper bound of optimum and prune unnecessary branches in the early stage [51], and thus decrease the computation time. The initial value is provided by iterated optimization of EV sharing system and power market clearing, as Algorithm 1 shows.

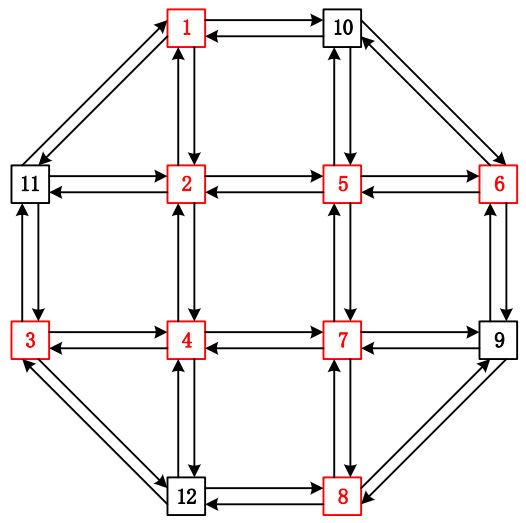

Fig. 1. The transportation network.

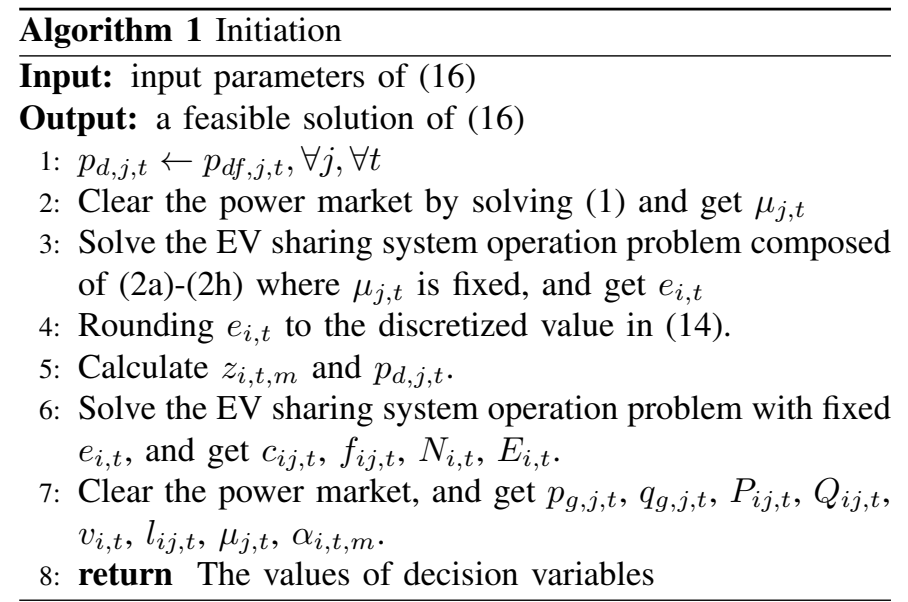

First, clear the power market without considering the load of EV sharing system, and get nodal prices. Then use these prices and solve the EV sharing system operation problem, which is composed of (2a)-(2h) where $\mu$ is fixed. After that, we get a solution of service pricing and charging scheduling, as well as charging load at nodes. Modify the solution and make it feasible for the discretized charging energy constraint (14). Then the power market is cleared again and nodal prices are renewed, and the solution can serve as an initial point.

In Algorithm 1, the market clearing problem is an SOCP, and the charging scheduling gives rise to a convex quadratic problem. Both of them can be efficiently solved. Nevertheless, such a solution may not be the global optimum from the perspective of the company.

\section{CASe Study}

\section{A. Settings}

We consider an EV sharing system on a transportation network with 12 nodes and 40 links shown in Fig. 1. There is a parking lot on each red node, and the EV sharing system possesses 8 parking lots in total, labeled from 1 to 8 in Fig. 1. Detailed data can be found at [52].

A day is divided into $T=24$ periods with length $\Delta t=$ $1 \mathrm{~h}$. The total traffic demand varies from period to period, causing different levels of congestions. We use classical traffic 
TABLE I

COEFFICIENT OF POWER LOAD.

\begin{tabular}{cccccc}
\hline Period & Coefficient & Period & Coefficient & Period & Coefficient \\
\hline 1 & 0.7240 & 9 & 0.9457 & 17 & 0.8597 \\
2 & 0.6787 & 10 & 0.8914 & 18 & 0.8914 \\
3 & 0.6425 & 11 & 0.8145 & 19 & 0.9593 \\
4 & 0.6878 & 12 & 0.7964 & 20 & 0.9321 \\
5 & 0.8371 & 13 & 0.7919 & 21 & 0.9231 \\
6 & 0.9864 & 14 & 0.8054 & 22 & 0.8914 \\
7 & 0.9955 & 15 & 0.8145 & 23 & 0.8145 \\
8 & 1.0000 & 16 & 0.8281 & 24 & 0.7330 \\
\hline
\end{tabular}

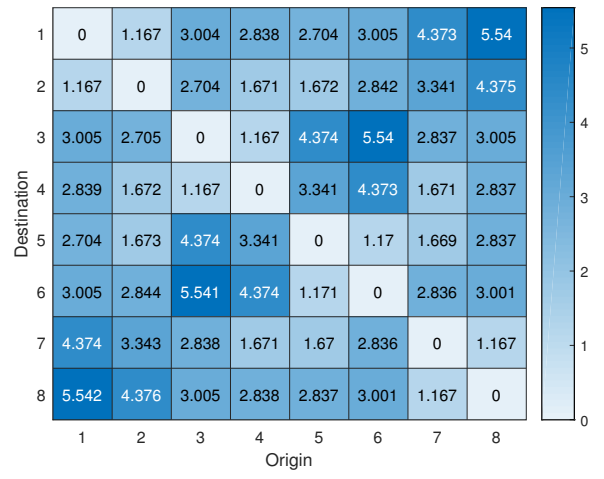

Fig. 2. The energy consumption data $L_{i j, 14}(\mathrm{kWh})$.

assignment model [53] to predict background traffic and road travel time. With this travel time information, shared EV users choose the fastest path (with shortest travel time) connecting their origin and destination. Energy consumption is a function of the length of the traveled distance and the road travel time. For instance, the power consumption data $L_{i j, 14}$ in period 14 is plotted in Fig. 2.

In the linear demand-price function (2b) of EV sharing clients, $a_{i j, t}$ can be regarded as the volume of potential clients, and $b_{i j, t}$ reflects the elasticity of the demand. We assume $a_{i j, t}$ is proportional to the value of O-D demand, with a maximal value of $\max _{t} \sum_{i} \sum_{j} a_{i j, t}=3000 ; b_{i j, t}=20$ is the same for any $i, j$ and $t$. Let $N_{i, 0}=100$ for all parking lots (this does not necessarily mean that there are 100 vehicles in each parking lot at the beginning of the first period). $E_{\max }=27$ $\mathrm{kWh}, E_{\min }=3 \mathrm{kWh}, \bar{N}_{i}=200, \bar{e}_{i}=2.5 \mathrm{MWh}, \bar{p}=0.25$ MWh and $E_{i, 0}=2.1 \mathrm{MWh}$ are used in our tests.

The IEEE 33-node test system is used as the distribution network, whose topology is shown in Fig. 3, and system data are consistent with Matpower [54]. Bus 0 is the slack bus, and the voltage magnitude is 1 p.u. The time-varying load is obtained by multiplying the nodal load with a time-varying parameter shown in Table I. Parking lot connections with the distribution grid are marked with red squares in Fig. 3. Let $\rho=$ $\$ 0.20 / \mathrm{kWh}$. Suppose there are gas-fired distributed generators at the nodes $4,9,14,19,24$ and 29, where the generation cost coefficients $\rho_{g}$ are $0.27,0.26,0.26,0.25,0.25$ and $0.28 \$ / \mathrm{kWh}$ respectively. The power output limits are $0 \leq p_{j}^{g} \leq 0.17$ and $-0.17 \leq q_{j}^{g} \leq 0.17$ in per unit for all units. The node voltage bound is $0.9^{2} \leq v_{i} \leq 1.1^{2}$ (because $v_{i}$ actually represents squared voltage magnitude), and the line current bound is $0 \leq$ $l_{i j} \leq 1.04$ in per unit.

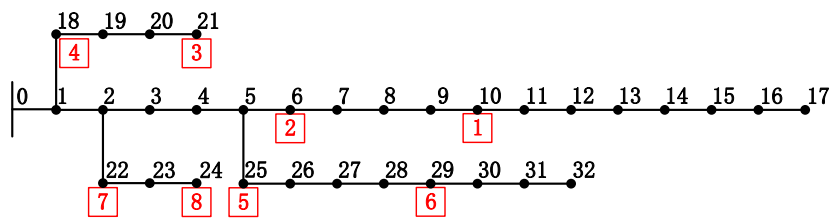

Fig. 3. The IEEE 33-node distribution network.

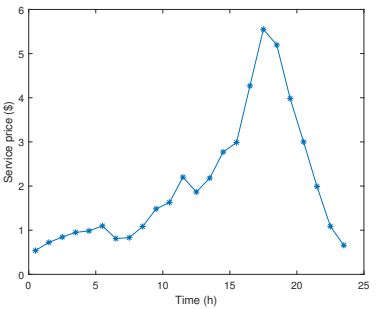

(a) The service price $c_{6,5, t}$.

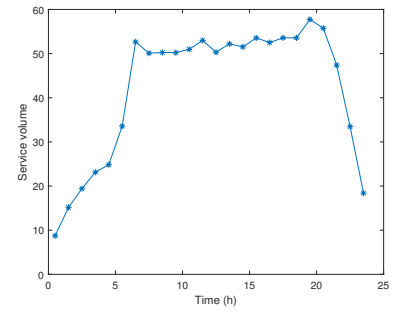

(b) The times of service $f_{6,5, t}$.
Fig. 4. Pricing result of travel from 6 to 5 in Case 1.

When we set up MIQP (16), $v=7, M=5$ and $N_{B}=8$ are used. The MIQP is coded in MATLAB environment using YALMIP [55], and solved by GUROBI 8.0.1 [56]. We slightly relax the equality constraint $E_{i, T}=E_{i, 0}$ to $E_{i, 0} \leq E_{i, T} \leq$ $E_{i, 0}+0.006$ p.u., aiming at providing a certain degree of flexibility. Since more stored energy makes the system more flexible in the second day, this relaxation is reasonable. The solver optimality gap for convergence is set to $1 \%$.

For comparison, we consider four cases. Case 1 is the benchmark case where the proposed method is used. Case 0 does not consider the EV sharing system (only OPF problem is solved). Case 2 employs a simple strategy to determine service price and charging schedule, which will be introduced later. Case 3 adopts the dual-value time-of-use price. The two values are determined from the average system LMP in Case 0 across the same periods. Case 4 utilizes real-time price for the 24 periods. In each period, the electricity price is set to the spatial average LMP in Case 0.

\section{B. Results of the benchmark case}

In the benchmark case, the computation time is $1021 \mathrm{~s}$, which is acceptable for a day-ahead application problem. The profit of the shared EV company is about $\$ 33975$. The service price $c_{i j, t}$ for any origin parking lot $i$, destination parking lot $j$ and period $t$ is also obtained. For example, the price curve between parking lots 6 and 5 and the volume of clients are plotted in Fig. 4a and Fig. 4b, respectively. The figure shows that the service price is time-varying, and a peak occurs at the evening rush hour.

In Case 1, the total volume of EV sharing service in each period $\left(\sum_{i} \sum_{j} f_{i j, t}\right)$ is shown in Fig. 5. It can be observed that almost all 800 vehicles owned by the company are in service from 7:00 a.m. to 8:00 p.m. Because of the price elasticity, no more demand arises and the profit of the company is maximized.

In Case 1, the volume of available EVs $N_{i, t}$ in each parking lot is plotted in Fig. 6a, and the average charging power 


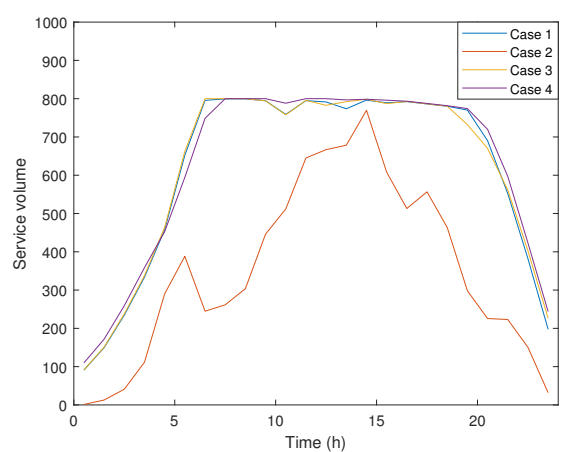

Fig. 5. The total service volume $\sum_{i} \sum_{j} f_{i j, t}$ in each period.

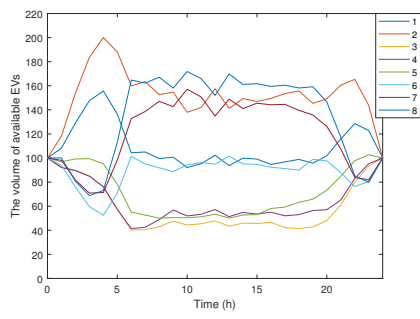

(a) The volume of available EVs.

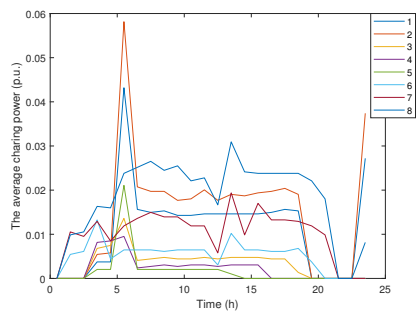

(b) The average charging power.
Fig. 6. The available EVs and charging demand at parking lots in Case 1.

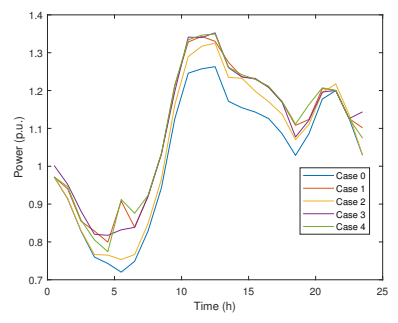

(a) The total power load.

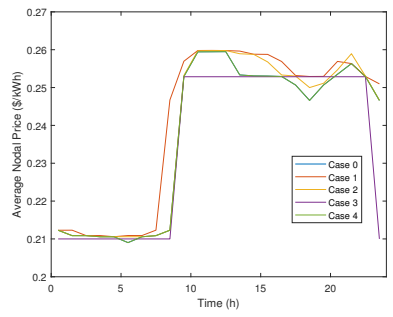

(b) The average nodal price.
Fig. 7. The total power load and average nodal price.

$e_{i, t} / \Delta t$ in Fig. 6b. EVs are traveling between parking lots. Their spatial distribution is changing over time, but the initial and final conditions are equal. A charging peak is found in period 6 , because the electricity price is low.

In Fig. 7a, the total charging load $\sum_{j} p_{j, t}^{d}$ is drawn. The curve associated with Case 0 represents the traditional load profile because EV charging is not considered in this case. It clearly shows that with the proposed method in Case 1, the minimum load during 03:00 to 07:00 grows larger compared to Case 0, although the maximum load also increases, as power load peak coincides with the traffic morning peak. The load peak-valley differences are 0.54 p.u. in Case 0 and Case 1 . Fig. $7 \mathrm{~b}$ shows the average nodal price which is defined as $\left(\sum_{j} \mu_{j, t} p_{d, j, t}\right) /\left(\sum_{j} p_{d, j, t}\right)$, illustrating that during night, the average price exhibits little difference, and price discrepancy occurs between 10 a.m. and 10 p.m. Compared with Case 0 , the charging demands elevate the electricity price in Case 1.

\section{Results of a simple strategy}

We compare the proposed method with a simple strategy of service pricing and charging scheduling established in Case 2. In this case, the service price from parking lot $i$ to $j$ is set to be a constant $c_{i j}^{\prime}$ for all time periods, and the EV sharing system can decide the service volume within the range of client demand. Specifically, let $c_{i j}^{\prime}$ be the average travelling price in Case 1, i.e., $c_{i j}^{\prime}=\left(\sum_{t} c_{i j, t} f_{i j, t}\right) /\left(\sum_{t} f_{i j, t}\right)$. This constant is related to the distance between the two parking lots, so it is reasonable.

For parking lot $i$ in period $t$, the demand of traveling to parking lot $j$ is $\max \left\{a_{i j, t}-b_{i j, t} c_{i j}^{\prime}, 0\right\}$, where the maximum is to avoid a negative demand. The available EVs are used for trips according to the demand. If $\sum_{k} \max \left\{a_{i k, t}-b_{i k, t} c_{i k}^{\prime}, 0\right\}>0$, let

$$
\begin{gathered}
f_{i j, t}=R_{i j, t} \cdot \min \left\{N_{i, t-1}, \sum_{k} \max \left\{a_{i k, t}-b_{i k, t} c_{i k}^{\prime}, 0\right\}\right\} \\
R_{i j, t}=\frac{\max \left\{a_{i j, t}-b_{i j, t} c_{i j}^{\prime}, 0\right\}}{\sum_{k} \max \left\{a_{i k, t}-b_{i k, t} c_{i k}^{\prime}, 0\right\}}
\end{gathered}
$$

The minimum in the first equation guarantees that $\sum_{j} f_{i j, t} \leq$ $N_{i, t-1}$, so the available EVs are adequate for the service. The number $R_{i j, t}$ is a proportionality coefficient reflecting the proportion of service between trips from $i$ to different parking lots. If $\sum_{k} \max \left\{a_{i k, t}-b_{i k, t} c_{i k}^{\prime}, 0\right\}=0$, let $f_{i j, t}=0$.

A greedy charging strategy is used: Charge EVs whenever they are not fully-charged in the parking lots. Then

$$
E_{i, t}=E_{\max } N_{i, t}, e_{i, t}=\sum_{j} L_{j i, t} f_{j i, t}
$$

For this simple strategy, constraints (2c)-(2f) hold, but the equal operating states constraint $(2 \mathrm{~g})$ and the upper bounds of $\mathrm{EV}$ volume and charging power in (2h) may not be necessarily true. Since there is no price regulation, parking lot daily balancing condition $(2 \mathrm{~g})$ is neglected.

The optimization problem in Case 2 can be solved in 28 s. The profit of Case 2 is $\$ 10897$, which is much lower than that in Case 1. The service volumes of Case 1 and Case 2 are compared in Fig. 5. In Case 2, EVs are not fully utilized in all periods. The load profile in Case 2 exhibits a deeper valley than that in Case 1 because of smaller service volume, as Fig. $7 \mathrm{a}$ shows. The peak-valley difference is 0.57 p.u. In Fig. 7b, the average nodal price of Case 2 is comparable to that of Case 1 in most time periods. However, at the end of the day, the volume of EVs at parking lots are about 132, 131, 44, 50, 65, 120, 188, 69 in Case 2, i.e., the parking lot usage is not self-balanced. Thus, the proposed strategy performs better than that in Case 2 in terms of economy and vehicle reallocation.

\section{Results of Fixed Prices}

Case 3 and Case 4 use fixed prices, i.e., the electricity price is an exogenous-given constant rather than a variable, regardless of the volume of demand. So the market clearing problem is unnecessary, and results can be obtained by independently solving the EV sharing system operation problem comprised of (2a)-(2h). 
TABLE II

RESULTS UNDER DIFFERENT $a_{i j, t}$.

\begin{tabular}{cccc}
\hline Multiple & Objective (\$) & Runtime (s) & $\sum_{i} \sum_{j} \sum_{t} f_{i j, t}$ \\
\hline 1.00 & 33975 & 1021 & 14763 \\
1.25 & 51564 & 1530 & 15929 \\
1.50 & 70017 & 1165 & 16722 \\
1.75 & 88928 & 1962 & 17323 \\
\hline
\end{tabular}

TABLE III

RESULTS UNDER DIFFERENT $b_{i j, t}$.

\begin{tabular}{cccc}
\hline$b_{i j, t}$ & Objective (\$) & Runtime (s) & $\sum_{i} \sum_{j} \sum_{t} f_{i j, t}$ \\
\hline 5 & 172550 & 1042 & 15995 \\
10 & 79949 & 1519 & 15662 \\
15 & 49172 & 2052 & 15234 \\
20 & 33975 & 1021 & 14763 \\
\hline
\end{tabular}

The price curves in Case 3 and Case 4 are shown in Fig. 7b. In Case 3 , the electricity price is $0.253 \$ / \mathrm{kWh}$ during periods 10:00-23:00 and 0.210\$/kWh during periods 0:00-9:00. The runtime of Case 3 and Case 4 is about 20 seconds. The peak charging power in the benchmark case is lower than those in Case 3 and Case 4, because a higher load generally leads to higher nodal prices, so the charging power cannot be too high. In the two cases with fixed electricity price, the price does not change even if the charging power increases, so the charging power is higher than that in the benchmark case. By-and-large, the charging power in Case 4 is closer to the benchmark case compared with Case 3 as shown in Fig. 7a, because of the more similar electricity price. The peak-valley difference is 0.57 p.u. in Case 4, which is larger than that of Case 1.

In summary, the proposed strategy brings more benefits than fixed pricing schemes from the grid operation perspective.

\section{E. Parameter Sensitivity Analysis}

Finally, we investigate the impact of demand elasticity parameter $a_{i j, t}$ on the results. Multiply $a_{i j, t}$ by a constant for all $i, j$, and $t$. The results are shown in Table II. As demand increases, the profit and the total service volume increase.

Table III shows the results under different $b_{i j, t}$. Larger $b_{i j, t}$ means the users are more sensitive to service price, so the profit and the service volume decrease as $b_{i j, t}$ increases. All tests in Table II and Table III can be solved within an hour.

\section{CONCLUSION}

This paper proposes optimal pricing and operation strategy for one-way EV sharing system. Considering EV mobility and market participation is the main feature of the proposed model. A bilevel model is set forth to capture the influence of EV charging on distribution system operation and LMP, and it is reformulated as a mixed-integer quadratic program through polyhedral approximation of second-order cones, primal-dual optimality condition, and product term linearization. Case studies show that the proposed method has several advantages. From the perspective of distribution system, the load valley is filled because some charging demand shifts to night when the electricity is cheaper; new demand peaks caused by EV charging is rarely seen because LMP provides an adaptive mechanism in response to the change of demand. From the perspective of transportation system, some traffic demand in rush hours may wish to switch to public transport because of the high service price of shared vehicles, which may help lessen traffic jams in big cities.

\section{REFERENCES}

[1] N. McCarthy. (2018) Electric car sales are surging in china [infographic]. [Online]. Available: https://www.forbes.com/sites/niallmccarthy/ 2018/06/01/electric-car-sales-are-surging-in-china-infographic/ \#1000a653d1f7

[2] S. Habib, M. M. Khan, F. Abbas, L. Sang, M. U. Shahid, and H. Tang, "A comprehensive study of implemented international standards, technical challenges, impacts and prospects for electric vehicles," IEEE Access, vol. 6, pp. 13 866-13890, 2018.

[3] N. Agatz, A. Erera, M. Savelsbergh, and X. Wang, "Optimization for dynamic ride-sharing: A review," Eur. J. Oper. Res., vol. 223, no. 2, pp. 295-303, Dec. 2012.

[4] S. Hu, P. Chen, H. Lin, C. Xie, and X. Chen, "Promoting carsharing attractiveness and efficiency: An exploratory analysis," Transport. Res. Part D-Transport. Environ., vol. 65, pp. 229-243, Dec. 2018.

[5] V. Nerenberg, M. Bernard, and N. E. Collins, "Evaluation results of san francisco bay area station-car demonstration," Transp. Res. Record, vol. 1666, no. 1, pp. 110-117, 1999.

[6] S. Shaheen, D. Sperling, and C. Wagner, "Carsharing in europe and north america: Past, present, and future," Transp. $Q$., vol. 52, no. 3, pp. 35-52, 1998.

[7] S. A. Shaheen and A. P. Cohen, "Carsharing and personal vehicle services: Worldwide market developments and emerging trends," Int. J. Sustain. Transp., vol. 7, no. 1, pp. 5-34, 2013.

[8] M. Bruglieri, A. Colorni, and A. Lu, "The vehicle relocation problem for the one-way electric vehicle sharing: an application to the milan case," in 16th Meeting of the Euro-Working-Group-in-Transportation, Porto, PORTUGAL, Sep. 2013, pp. 18-27.

[9] A. G. Kek, R. L. Cheu, Q. Meng, and C. H. Fung, "A decision support system for vehicle relocation operations in carsharing systems," Transp. Res. Pt. e-Logist. Transp. Rev., vol. 45, no. 1, pp. 149-158, Jan. 2009.

[10] A. D. Febbraro, N. Sacco, and M. Saeednia, "One-way carsharing: Solving the relocation problem," Transp. Res. Record, vol. 2319, no. 1, pp. 113-120, 2012.

[11] A. Angelopoulos, D. Gavalas, C. Konstantopoulos, D. Kypriadis, and G. Pantziou, "Incentivized vehicle relocation in vehicle sharing systems," Transp. Res. Pt. C-Emerg. Technol., vol. 97, pp. 175-193, Dec. 2018.

[12] L. He, H. Mak, Y. Rong, and Z. Shen, "Service region design for urban electric vehicle sharing systems," M\&SOM-Manuf. Serv. Oper. Manag., vol. 19, no. 2, pp. 309-327, 2017.

[13] M. Honarmand, A. Zakariazadeh, and S. Jadid, "Optimal scheduling of electric vehicles in an intelligent parking lot considering vehicle-to-grid concept and battery condition," Energy, vol. 65, pp. 572-579, Feb. 2014.

[14] M. S. Kuran, A. C. Viana, L. Iannone, D. Kofman, G. Mermoud, and J. P. Vasseur, "A smart parking lot management system for scheduling the recharging of electric vehicles," IEEE Trans. Smart Grid, vol. 6, no. 6, pp. 2942-2953, Nov. 2015.

[15] M. Yazdani-Damavandi, M. P. Moghaddam, M. Haghifam, M. ShafieKhah, and J. P. S. Catalao, "Modeling operational behavior of plug-in electric vehicles' parking lot in multienergy systems," IEEE Trans. Smart Grid, vol. 7, no. 1, pp. 124-135, Jan. 2016.

[16] Y. Guo, J. Xiong, S. Xu, and W. Su, "Two-stage economic operation of microgrid-like electric vehicle parking deck," IEEE Trans. Smart Grid, vol. 7, no. 3, pp. 1703-1712, May 2016.

[17] R. F. Atallah, C. M. Assi, W. Fawaz, M. H. K. Tushar, and M. J. Khabbaz, "Optimal supercharge scheduling of electric vehicles: Centralized versus decentralized methods," IEEE Trans. Veh. Technol., vol. 67, no. 9, pp. 7896-7909, Sep. 2018.

[18] N. Neyestani, M. Y. Damavandi, M. Shafie-Khah, J. Contreras, and J. P. S. Catalo, "Allocation of plug-in vehicles' parking lots in distribution systems considering network-constrained objectives," IEEE Trans. Power Syst., vol. 30, no. 5, pp. 2643-2656, Sep. 2015.

[19] M. Shafie-Khah, E. Heydarian-Forushani, G. J. Osorio, F. A. S. Gil, J. Aghaei, M. Barani, and J. P. S. Catalao, "Optimal behavior of electric vehicle parking lots as demand response aggregation agents," IEEE Trans. Smart Grid, vol. 7, no. 6, pp. 2654-2665, Nov. 2016. 
[20] N. Neyestani, M. Y. Damavandi, M. Shafie-khah, A. G. Bakirtzis, and J. P. S. Catalo, "Plug-in electric vehicles parking lot equilibria with energy and reserve markets," IEEE Trans. Power Syst., vol. 32, no. 3, pp. 2001-2016, May 2017.

[21] M. Shafie-Khah, P. Siano, D. Z. Fitiwi, N. Mahmoudi, and J. P. S. Catalo, "An innovative two-level model for electric vehicle parking lots in distribution systems with renewable energy," IEEE Trans. Smart Grid, vol. 9, no. 2, pp. 1506-1520, 2018.

[22] C. Rottondi, G. Neglia, and G. Verticale, "Complexity analysis of optimal recharge scheduling for electric vehicles," IEEE Trans. Veh. Technol., vol. 65, no. 6, pp. 4106-4117, Jun. 2016.

[23] V. Monteiro, J. G. Pinto, and J. L. Afonso, "Operation modes for the electric vehicle in smart grids and smart homes: Present and proposed modes," IEEE Trans. Veh. Technol., vol. 65, no. 3, pp. 1007 - 1020, Mar. 2016.

[24] M. Bayati, M. Abedi, G. Gharehpetian, and et al., "Short-term interaction between electric vehicles and microgrid in decentralized vehicle-to-grid control methods," Prot. Control Mod. Power Syst., vol. 4:5, Feb. 2019.

[25] H. Turker and S. Bacha, "Optimal minimization of plug-in electric vehicle charging cost with vehicle-to-home and vehicle-to-grid concepts," IEEE Trans. Veh. Technol., vol. 67, no. 11, pp. 10281-10292, Nov. 2018.

[26] Y. Cheng and C. Zhang, "Configuration and operation combined optimization for EV battery swapping station considering PV consumption bundling," Prot. Control Mod. Power Syst., vol. 2:26, 2017.

[27] Y. J. Jang, "Survey of the operation and system study on wireless charging electric vehicle systems," Transportation Research Part C: Emerging Technologies, vol. 95, pp. 844-866, 2018.

[28] R. Riemann, D. Z. W. Wang, and F. Busch, "Optimal location of wireless charging facilities for electric vehicles: Flow-capturing location model with stochastic user equilibrium," Transportation Research Part C: Emerging Technologies, vol. 58, pp. 1-12, 2015.

[29] C. Ou, H. Liang, and W. Zhuang, "Investigating wireless charging and mobility of electric vehicles on electricity market," IEEE Trans. Ind. Electron., vol. 62, no. 5, pp. 3123-3133, May 2015.

[30] S. D. Manshadi, M. E. Khodayar, K. Abdelghany, and H. ster, "Wireless charging of electric vehicles in electricity and transportation networks," IEEE Trans. Smart Grid, vol. 9, no. 5, pp. 4503-4512, Sep. 2018.

[31] C. Yang, W. Lou, J. Yao, and S. Xie, "On charging scheduling optimization for a wirelessly charged electric bus system," IEEE Trans. Intell. Transp. Syst., vol. 19, no. 6, pp. 1814-1826, 2018.

[32] M. Parkin, Economics. Essex: Pearson Education UK, 2013.

[33] D. Jorge, G. Molnar, and G. Correia, "Trip pricing of one-way stationbased carsharing networks with zone and time of day price variations," Transportation Research Part B: Methodological, vol. 81, pp. 461 - 482, 2015.

[34] D. J. Graham and S. Glaister, "Road traffic demand elasticity estimates: A review," Transport Reviews, vol. 24, no. 3, pp. 261-274, 2004.

[35] M. Xu, Q. Meng, and Z. Liu, "Electric vehicle fleet size and trip pricing for one-way carsharing services considering vehicle relocation and personnel assignment," Transportation Research Part B: Methodological, vol. 111, pp. $60-82,2018$.

[36] M. Farivar and S. H. Low, "Branch flow model: Relaxations and convexification-part i," IEEE Trans. Power Syst., vol. 28, no. 3, pp. 2554-2564, Aug. 2013.

[37] S. H. Low, "Convex relaxation of optimal power flow part I: Formulations and equivalence," IEEE Trans. Control Network Systems, vol. 1, no. 1, pp. 15-27, Mar. 2014.

[38] S. Huang, Q. Wu, J. Wang, and H. Zhao, "A sufficient condition on convex relaxation of ac optimal power flow in distribution networks," IEEE Trans. Power Syst., vol. 32, no. 2, pp. 1359-1368, Mar. 2017.

[39] B. Stott, J. Jardim, and O. Alsac, "Dc power flow revisited," IEEE Trans. Power Syst., vol. 24, no. 3, pp. 1290-1300, Aug. 2009.

[40] M. E. Baran and F. F. Wu, "Network reconfiguration in distribution systems for loss reduction and load balancing," IEEE Trans. Power Deliv., vol. 4, no. 2, pp. 1401-1407, 1989.

[41] H. Yeh, D. F. Gayme, and S. H. Low, "Adaptive var control for distribution circuits with photovoltaic generators," IEEE Trans. Power Syst., vol. 27, no. 3, pp. 1656-1663, Aug. 2012.

[42] Y. Liu, J. Li, and L. Wu, "Distribution system restructuring: Distribution lmp via unbalanced acopf," IEEE Trans. Smart Grid, vol. 9, no. 5, pp. 4038-4048, Sep. 2018.

[43] Z. Yuan, M. R. Hesamzadeh, and D. R. Biggar, "Distribution locational marginal pricing by convexified acopf and hierarchical dispatch," IEEE Trans. Smart Grid, vol. 9, no. 4, pp. 3133-3142, 2018.
[44] H. Jiang and D. Ralph, "Smooth SQP methods for mathematical programs with nonlinear complementarity constraints," SIAM J. Optim., vol. 10 , no. 3, pp. 779-808, 2000.

[45] J. Fortuny-Amat and B. McCarl, "A representation and economic interpretation of a two-level programming problem," J. Oper. Res. Soc., vol. 32, no. 9, pp. 783-792, Sep. 1981.

[46] A. Ben-Tal and A. Nemirovski, "On polyhedral approximations of the second-order cone," Math. Oper. Res., vol. 26, no. 2, pp. 193-205, May 2001.

[47] R. Fernndez-Blanco, J. M. Arroyo, and N. Alguacil, "On the solution of revenue- and network-constrained day-ahead market clearing under marginal pricingpart i: An exact bilevel programming approach," IEEE Trans. Power Syst., vol. 32, no. 1, pp. 208-219, Jan. 2017.

[48] S. D. Manshadi and M. E. Khodayar, "Expansion of autonomous microgrids in active distribution networks," IEEE Trans. Smart Grid, vol. 9, no. 3, pp. 1878-1888, May 2018.

[49] H. Haghighat and B. Zeng, "Bilevel conic transmission expansion planning," IEEE Trans. Power Syst., vol. 33, no. 4, pp. 4640-4642, 2018.

[50] M. V. Pereira, S. Granville, M. H. C. Fampa, R. Dix, and L. A. Barroso, "Strategic bidding under uncertainty: A binary expansion approach," IEEE Trans. Power Syst., vol. 20, no. 1, pp. 180-188, Feb. 2005.

[51] M. Conforti, G. Cornuejols, and G. Zambelli, Integer programming. Cham Switzerland ; New York : Springer, 2014.

[52] [Online]. Available: https://github.com/xieruijx/EV-sharing

[53] Y. Sheffi, Urban transportation networks: equilibrium analysis with mathematical programming methods. New Jersey: Prentice Hall, 1985.

[54] R. D. Zimmerman, C. E. Murillo-Sanchez, and R. J. Thomas, "Matpower: Steady-state operations, planning, and analysis tools for power systems research and education," IEEE Trans. Power Syst., vol. 26, no. 1, pp. 12-19, Feb. 2011

[55] J. Löfberg, "YALMIP : A toolbox for modeling and optimization in MATLAB," in Proceedings of the IEEE International Symposium on Computer-Aided Control System Design, Taipei, Taiwan, China, Sep. 2004, pp. 284-289.

[56] Gurobi Optimization. (2019) Gurobi optimization - the stateof-the-art mathematical programming solver. [Online]. Available: http://www.gurobi.com/

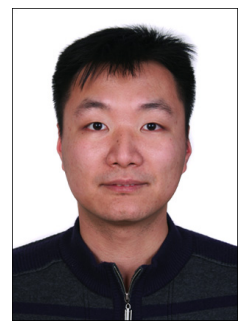

Wei Wei (SM'18) received the B.Sc. and Ph.D. degrees in electrical engineering from Tsinghua University, Beijing, China, in 2008 and 2013, respectively.

He was a Postdoctoral Research Associate with Tsinghua University from 2013 to 2015. He was a Visiting Scholar with Cornell University, Ithaca, NY, USA, in 2014, and with Harvard University, Cambridge, MA, USA, in 2015. He is currently an Associate Professor with Tsinghua University. His research interests include applied optimization and energy system economics.

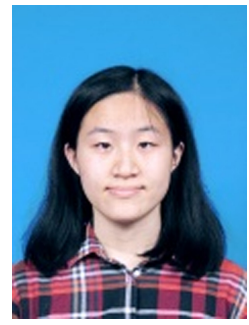

Rui Xie received the B.Sc. degree in electrical engineering and the B.Sc. degree in mathematics from Tsinghua University, Beijing, China, in 2017, where she is currently pursuing the Ph.D. degree with the Department of Electrical Engineering.

Her current research interests include optimization problems in the interdependent electrical power and transportation systems. 


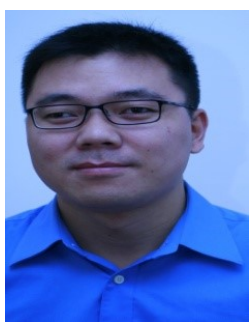

Qiuwei Wu (M'08-SM'15) obtained the B. Eng. and M. Eng. in Power System and Its Automation from Nanjing University of Science and Technology, Nanjing, China, in 2000 and 2003, respectively. $\mathrm{He}$ obtained the PhD degree in Power System Engineering from Nanyang Technological University, Singapore, in 2009.

He was a senior R\&D engineer with VESTAS Technology R\&D Singapore Pte Ltd from Mar. 2008 to Oct. 2009. He has been working at Department of Electrical Engineering, Technical University of Denmark (DTU) since Nov. 2009 (PostDoc Nov. 2009-Oct. 2010, Assistant Professor Nov. 2010-Aug. 2013, Associate Professor since Sept. 2013). He was a visiting scholar at Department of Industrial Engineering \& Operations Research (IEOR), University of California, Berkeley, from Feb. 2012 to May 2012 funded by Danish Agency for Science, Technology and Innovation (DASTI), Denmark. He was a visiting professor named by Y. Xue, an Academician of Chinese Academy of Engineering, at Shandong University, China, from Nov. 2015 to Oct. 2017. He was a visiting scholar at School of Engineering and Applied Science, Harvard University in 2018.

His research area is power system operation and control with high renewables, including wind power modeling and control, active distribution networks, and integrated energy systems. He is an Editor of IEEE Transactions on Smart Grid and IEEE Power Engineering Letters. He is also an Associate Editor of International Journal of Electrical Power and Energy Systems, Journal of Modern Power Systems and Clean Energy, IET Renewable Power Generation, and IET Generation, Transmission \& Distribution.

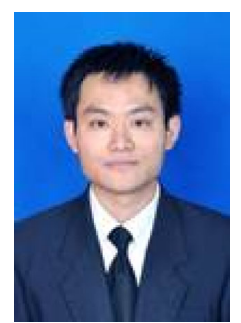

Tao Ding (SM'19) received the B.S.E.E. and M.S.E.E. degrees from Southeast University, Nanjing, China, in 2009 and 2012, respectively, and the Ph.D. degree from Tsinghua University, Beijing, China, in 2015. During 2013 and 2014.

$\mathrm{He}$ is currently an Associate Professor with the State Key Laboratory of Electrical Insulation and Power Equipment, School of Electrical Engineering, Xi' an Jiaotong University, Xi'an, China. He was a Visiting Scholar with the Department of Electrical Engineering and Computer Science, University of Tennessee, Knoxville, TN, USA. He has authored or coauthored more than 60 technical papers and authored by "Springer Theses" recognizing outstanding $\mathrm{Ph} . \mathrm{D}$. research around the world and across the physical sciences-Power System Operation With Large Scale Stochastic Wind Power Integration. His current research interests include electricity markets, power system economics and optimization methods, and power system planning and reliability evaluation.

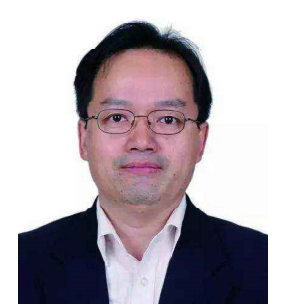

Shengwei Mei (F'15) received the B.Sc. degree in mathematics from Xinjiang University, Urumqi, China, the M.Sc. degree in operations research from Tsinghua University, Beijing, China, and the Ph.D. degree in automatic control from Chinese Academy of Sciences, Beijing, China, in 1984, 1989, and 1996, respectively.

$\mathrm{He}$ is currently a Professor of Tsinghua University, Beijing, China. His research interests include power system complexity and control, game theory and its application in power systems. 